\title{
PROSPECTIVE CLINICAL STUDY ABOUT THE PROGNOSIS IN EXTRAHEPATIC BILIARY ATRESIA PATIENTS FOLLOWING KASAI'S PORTOENTEROSTOMY
}

\author{
${ }^{1}$ Associate Professor, Department of Paediatric Surgery, Niloufer Hospital, Hyderabad. \\ ${ }^{2}$ Assistant Professor, Department of Paediatric Surgery, Niloufer Hospital, Hyderabad. \\ ${ }_{3}^{3}$ HOD, Department of Paediatric Surgery, Niloufer Hospital, Hyderabad. \\ ${ }_{4}^{4}$ Assistant Professor, Department of Paediatric Surgery, Niloufer Hospital, Hyderabad. \\ ${ }_{5}^{5}$ Assistant Professor, Department of Paediatric Surgery, Niloufer Hospital, Hyderabad.
}

Nagarjuna Kumbah', Gulam Mohammed Irfan², Ramesh Reddy Kota ${ }^{3}$, Srirampur Srinivas ${ }^{4}$, Hari Prasad Sharab

\section{ABSTRACT}

\section{PURPOSE}

To evaluate the prognosis in extrahepatic biliary atresia patients following Kasai's portoenterostomy.

\section{MATERIALS AND METHODS}

This prospective study of 64 Extrahepatic Biliary Atresia cases was conducted over 5 yrs. at Niloufer Hospital from March 2008 to March 2013. Of these, 43 patients underwent Kasai's procedure. Patients were analysed into 3 groups based on surgical outcome. A. Satisfactory response with Sr. Bilirubin $<2 \mathrm{mg} \%$ with isotope scan showing complete excretion of tracer; B. Partial response with Sr. Bilirubin $>2 \mathrm{mg} \%$; C. Therapeutic failure same as preoperative status.

\section{RESULTS}

9 (21\%) of the 43 cases following Kasai's portoenterostomy had satisfactory response without periportal fibrosis on liver biopsy. Of these 4 satisfactory responders presented within 2 months of age. The partial responders and therapeutic failure cases (79\%) had cirrhosis. Changes in postoperative bilirubin level is a good indicator of bile drainage.

\section{CONCLUSIONS}

The study revealed that early surgical intervention indicated by the absence of periportal fibrosis is a good prognostic indicator. The present series also emphasizes the need for regular followup, at least for a period of 5 years following portoenterostomy. By this, patient can be prognosticated and thereby refer the partial and therapeutic failure patients for early liver transplant program.

\section{KEYWORDS}

Biliary Atresia, Kasai's Portoenterostomy, Postoperative Outcome, Bilirubin, Liver Function.

HOW TO CITE THIS ARTICLE: Kumbah N, Irfan GM, Kota RR, et al. Prospective clinical study about the prognosis in extrahepatic biliary atresia patients following Kasai's portoenterostomy. J. Evolution Med. Dent. Sci. 2016;5(51):3333-3336, DOI: $10.14260 /$ jemds/2016/771

\section{INTRODUCTION}

Biliary atresia is a neonatal cholestatic disease characterized by progressive obliteration or discontinuity of the extrahepatic biliary system resulting in obstruction to bile flow. This disorder represents the most common surgically treatable cause of cholestasis encountered during the newborn period

Extrahepatic biliary atresia is not only the commonest cause of surgical jaundice in the new born period, but also one of the commonest indication for liver transplantation in paediatric age group. ${ }^{1}$ Liver transplantation is a preferred procedure for biliary atresia, but it has its own drawbacks if done in infants. The disadvantages include availability of primary donor, morbidity associated with transplantation like infection, oncogenesis and associated mortality.

Financial or Other, Competing Interest: None.

Submission 21-05-2016, Peer Review 06-06-2016,

Acceptance 09-06-2016, Published 27-06-2016.

Corresponding Author:

Nagarjuna Kumbah,

Associate Professor,

Department of Paediatric Surgery,

4-7-12/53; Ravindra Nagar Colony,

Nacharam, Hyderabad-500076,

India.

E-mail:nags_arjun@yahoo.co.in

DOI: 10.14260/jemds/2016/771
Kasai's portoenterostomy. ${ }^{2}$ is used as initial procedure in the treatment of biliary atresia. Dr. Kasai in 1959 has devised hepatic portoenterostomy and there have been encouraging results in treating this disease. Following Kasai portoenterostomy, a series of modified Kasai's procedures to increase bile drainage, irrigate the hepatic hilum and to prevent ascending cholangitis were subsequently attempted. However, the original Kasai's hepatic portoenterostomy remains the main choice for surgical intervention. Long-term prognosis is poor even with successful portoenterostomy because of ongoing liver damage at the level of hepatocyte. Clearance of jaundice (Less than $2 \mathrm{mg} / \mathrm{dL}$ or $34 \mathrm{mmol} / \mathrm{L}$ ) after Kasai has been reported to be around $60 \%, 3,4$

Following hepatic portoenterostomy, patient can be either cured with bilirubin level less than $2 \mathrm{mg} / \mathrm{dl}$, have a partial response or result in therapeutic failure. This prospective study focuses on identifying patients with partial response and therapeutic failure by a close followup for 5 years to prognosticate and refer them for liver transplantation. The study done at Niloufer Hospital for Women and Children from March 2008 to March 2013 included 64 cases of biliary atresia, out of which 43 underwent Kasai's portoenterostomy. These cases were evaluated to note post-operative bile drainage revealed by changes in Sr. Bilirubin levels, developmental assessment, pruritus and to note the episodes of cholangitis. 


\section{MATERIALS AND METHODS}

This prospective study was conducted at Niloufer Hospital for women and children from March 2008 to March 2013 to evaluate the prognosis in patients who underwent Kasai portoenterostomy for biliary atresia.

The study included 64 cases of extrahepatic biliary atresia, of these 43 patients underwent Kasai procedure. Liver biopsy and flushing of biliary tract was done in remaining 21 patients. These patients were evaluated by noting clinical history including age at the time of procedure, liver function test; ultrasound abdomen and isotope scan study. Mean age at time of surgery in our study was $66+/-20$ days. Coagulation abnormalities were corrected and patients were posted for surgery after informed consent.

Under general anaesthesia in supine position, a small right sub-costal incision was made and gallbladder identified and examined for patency. If gallbladder is distended, stay sutures were taken and per-operative cholangiogram was done. If the gallbladder was atretic, without a lumen, the incision was extended and Kasai procedure was performed. Porta was dissected; fibrous cone anterior to the bifurcation of portal vein was dissected and transected. Bifurcation of portal vein into right and left branches was taken as landmark of transection of fibrous cone. Porto-intestinal continuity was restored with Roux en-Y jejunal anastomosis.

The intra-operative findings observed at the time of surgery include status of liver, gallbladder, biliary tracts, portal plate, ascites and bile drainage after dissection of portal plate, presence of collaterals and splenomegaly. Liver biopsy was sent for histopathological examination. The pathologists at the Institute for Women and Child Health Niloufer Hospital have classified the changes in biliary atresia as early and late changes. The early changes include portal oedema, ductular proliferation and inspissated bile in ductules. The late changes include periportal fibrosis with irregularity of lining cholangiocytes of the intrahepatic ducts represented by vacuolization, nuclear pyknosis, infiltration by inflammatory cells, thickening, progressive atrophy and disappearance of ducts.

In the post-operative period patients were treated with steroid for 6 weeks, choleretic agent ursodeoxycholic acid and were supplemented with Vitamin-A and Vitamin-D. After surgery, patients were followed up at monthly intervals for first 3 months for one year, then once in every 3 months for 5 years to monitor the progress. During followup various parameters that were noted include detailed history, clinical examination and laboratory investigations.

The clinical history includes presence of jaundice, episodes of cholangitis, stool colour, urine colour, pruritus and bleeding episodes. Cholangitis was defined by presence of high-grade fever with jaundice and acholic stools and were treated with antibiotics and supportive treatment. The bleeding episodes were considered significant if patients had developed haematemesis, melena or purpura. Technetium 99 Mebrofenin hepatobiliary scan was done in patients with satisfactory response at 6 months after surgery.

The laboratory investigations include serial liver function tests, prothrombin time, ultrasound abdomen and isotope study. Liver function tests include serial total bilirubin, conjugated and unconjugated bilirubin, AlanineAminotransferase (ALT), Aspartate Aminotransferase (AST) and alkaline phosphatase.
The radiological investigations include ultrasonography of abdomen to note echogenicity and irregularity of liver, intrahepatic biliary dilatation or formation of cyst and evidence of portal hypertension.

The observation and results were statistically analysed. The observations were focused on certain prognostic indicators, which include patient age at the time of surgery, type of atresia, presence of bile flow at the time of surgery, presence of liver cirrhosis, liver function with regard to bilirubin level and number of episodes of post-operative cholangitis.

Patients were analysed and grouped into three categories according to the outcome. These include cases with satisfactory response, partial response and therapeutic failure. Satisfactory response includes patients with serum bilirubin less than $2 \mathrm{mg} / \mathrm{dL}$ with isotope scan showing complete clearance of tracer in 60 minutes. Partial response includes patients with serum bilirubin greater than $2 \mathrm{mg}$ with some biliary drainage. Therapeutic failure includes patient outcome same as pre-operative status or with further deterioration.

\section{STATISTICAL ANALYSIS}

The mean values of liver function tests preoperatively and postoperatively at 3 months, 3 to 6 months and after 6 months on followup after portoenterostomy were calculated along with the standard error. The $\mathrm{p}$ value was calculated by the paired $T$ test. $P$ value $<0.05$ was considered as significant.

\section{RESULTS}

In this study group, male-to-female ratio was $3: 2$. The mean age of the patient at the time of surgery was $66+/-20$ days and was significantly higher in partial and therapeutic failure groups. Only 4 patients who underwent surgery within 2 months of age were jaundice free with bilirubin less than 2 $\mathrm{mg} / \mathrm{dL}$.

Of the 43 cases of biliary atresia following Kasai portoenterostomy, 40 were type 3 (93\%), 2 (4.6\%) were type 2 and 1 patient $(2.3 \%)$ had type I lesion. The intraoperative findings include atretic gallbladder with fibrosed portal plate in 40 patients (93\%). Bile flow was seen after portal plate dissection in 35 patients (81.3\%). Of these, only nine patients (21\%) had satisfactory response with serum bilirubin less than $2 \mathrm{mg} / \mathrm{dL}$. Irregularity or nodularity of liver was seen in 25 patients $(58.1 \%)$ and showed cirrhosis on histopathological examination.

The liver biopsy had shown early changes of biliary atresia in 13 patients (30.2\%). Among these, 9 had satisfactory response and four had partial response. Six patients (13.9\%) with partial response and 24 patients (55.8\%) of therapeutic failure showed late changes of biliary atresia. Postoperatively, four patients had bilirubin $<2 \mathrm{mg} \%$ within 3 months and 9 cases within 6 months with acholic stools; 4 patients with partial response and 6 with therapeutic failure had pruritus within six months following Kasai portoenterostomy.

During the follow-up period after surgery, 5 patients within 3 months and 6 patients within 6 months of satisfactory response developed cholangitis. Among the partial response patients, one patient within 3 months and 4 within six months developed cholangitis. On contrary cholangitis was not seen in therapeutic failure patients following hepaticoportoenterostomy. Bleeding episodes were seen in 4 
patients with therapeutic failure, 2 within 3 months and 2 after 6 months following surgery.

There was high mortality among the therapeutic failure patients; 20 of the 24 patients with therapeutic failure expired during the follow-up. Of these, 3 patients expired within 3 months following surgery. The causes in these patients were hepatic failure, internal bleeding and intestinal obstruction.

The other clinical parameter monitored following hepaticoportoenterostomy was psychomotor development. It was observed that nine patients with satisfactory response showed normal psychomotor development and normal weight gain. Psychomotor development is delayed in partial and therapeutic failure cases.

The postoperative ultrasound of abdomen showed normal appearance of liver in 6 patients of the satisfactory group. While 3 patients showed mild increased echogenicity of liver. The partial response and therapeutic failure patients showed increased echogenicity, irregularity, nodularity, intrahepatic cyst formation and portosystemic collaterals suggestive of portal hypertension.

\begin{tabular}{|c|c|c|c|c|c|c|c|c|}
\hline $\begin{array}{c}\text { Liver Function } \\
\text { Tests }\end{array}$ & \multicolumn{2}{|c|}{$\begin{array}{c}\text { Total } \\
\text { Bilirubin }\end{array}$} & \multicolumn{2}{c|}{$\begin{array}{c}\text { Direct } \\
\text { Bilirubin }\end{array}$} & \multicolumn{2}{c|}{$\begin{array}{c}\text { Alanine } \\
\text { Transaminase }\end{array}$} & $\begin{array}{c}\text { Alkaline } \\
\text { Phosphatase }\end{array}$ \\
\hline $\begin{array}{c}\text { Response } \\
\text { Group }\end{array}$ & Pre-op & Post-op & Pre-op & Post-op & Pre-op & Post-op & Pre-op & Post-op \\
\hline $\begin{array}{c}\text { Satisfactory } \\
\text { Response }\end{array}$ & $13.8+/-1.04$ & $1.34+/-0.11$ & $6.64+/-0.56$ & $0.58+/-0.06$ & $324+/-21$ & $67+/-6$ & $401+/-22$ & $88+/-6$ \\
\hline $\begin{array}{c}\text { Partial } \\
\text { Response }\end{array}$ & $16.5+/-1.02$ & $5.7+/-0.4$ & $7.17+/-0.45$ & $2.2+/-0.27$ & $321+/-33$ & $324+/-15$ & $396+/-12$ & $348+/-25$ \\
\hline $\begin{array}{c}\text { Therapeutic } \\
\text { Failure }\end{array}$ & $15.9+/-0.72$ & $16.9+/-0.35$ & $7.12+/-0.44$ & $7.27+/-0.20$ & $313+/-18$ & $395+/-12$ & $357+/-24$ & $400+/-28$ \\
\hline \multicolumn{8}{|c|}{ Table 1: Comparison of Liver Function Tests in Various Outcome Groups } \\
\hline
\end{tabular}

Note. Values expressed in mean_SE of mean; Abbreviations: $\quad$ Pre-op=preoperative; postoperative.)

In this study, there was a significant decrease in the mean preoperative bilirubin level from $13.8+/-1.04$ to post-op $1.34+/-0.11 \mathrm{mg} / \mathrm{dL}$ at 6 months of follow-up period in the satisfactory group ( $\mathrm{p}$ value less than 0.05 ). This is reflected in the direct serum bilirubin levels in a similar pattern. The partial response patients showed decrease in mean bilirubin levels at 6 months of follow-up period. (Mean pre-op bilirubin level: $16.5+/-1.02$, postop bilirubin levels $5.7+/-0.4$ ). On contrary, the therapeutic failure patient showed mild increase in bilirubin levels.

Present study showed significant decrease in postoperative alanine aminotransaminases in satisfactory group at (Pre-op and post-op levels). However, the partial and therapeutic patients had persistently elevated levels. Similarly, alkaline phosphatase decreased in satisfactory group in comparison to partial and therapeutic failure. The preop alkaline phosphatase is $357+/-24$, the postop alkaline phosphatase $400+/-28$.

\begin{tabular}{|c|c|c|c|c|}
\hline \multirow[b]{2}{*}{ Prognostic Factor } & & \multicolumn{3}{|c|}{ No. of Patients } \\
\hline & & $\begin{array}{c}\text { Satisfactory Response } \\
9(21 \%) \\
\end{array}$ & $\begin{array}{c}\text { Partial Response } 10 \\
(23.2 \%)\end{array}$ & $\begin{array}{c}\text { Therapeutic Failure } \\
24(55.8 \%)\end{array}$ \\
\hline \multirow{3}{*}{$\begin{array}{c}\text { Age at the Time of } \\
\text { Surgery }\end{array}$} & 45-60 days & 4 & 0 & 0 \\
\hline & 60-90 days & 5 & 4 & 4 \\
\hline & $>90$ days & 0 & 6 & 20 \\
\hline \multirow{3}{*}{ Type of Atresia } & Type 1 & 1 & 0 & 0 \\
\hline & Type 2 & 2 & 0 & 0 \\
\hline & Type 3 & 6 & 10 & 24 \\
\hline \multirow{2}{*}{$\begin{array}{c}\text { Status of Liver } \\
\text { (Histopathology Findings) }\end{array}$} & Early changes & 9 & 4 & 0 \\
\hline & Late changes & 0 & 6 & 24 \\
\hline \multicolumn{5}{|c|}{ Table 2: Prognostic Factors Following Hepatic Portoenterostomy } \\
\hline
\end{tabular}

In this study, the main prognostic factors which influenced the outcome include age at the time of surgery, type of biliary atresia and presence or absence of liver cirrhosis. It is observed in this study that the patients with satisfactory biliary drainage will develop cholangitis. There were no episodes of cholangitis in patients with therapeutic failure.

\section{Outcome following Kasai's Portoenterostomy}

The present series showed $21 \%$ patients in satisfactory group, while the partial and therapeutic failure includes 23.2 and $55.8 \%$ respectively.

\section{DISCUSSION}

Biliary atresia, an obliterative cholangiopathy is a progressive neonatal disorder. It is fatal if left untreated. The sequelae of untreated biliary atresia include progressive cholestasis, cirrhosis, portal hypertension and hepatic failure. To stop the sequel, it is important to open up the small biliary ductules at portahepatis. This is achieved to some extent by Kasai hepatic portoenterostomy and also delay the time interval for liver transplantation.

The average male-to-female sex ratio in the present series is 3:2. A similar male predominance was noted by V. Bhatnagar et $\mathrm{al}^{5}$ and Saket et al. $^{6}$ This is in contrast to female predominance seen with other studies. The mean age of surgery for the biliary atresia in present series is $66+/-20$ days. This is an important prognostic factor, which influences the outcome in biliary atresia. In this study only 4 patients 
within 2 months of age were anicteric following surgery. Patient presenting after 2 months of age showed.

It is well documented by various studies that early surgical restoration of biliary drainage has good outcome. $6,7,893 \%$ of biliary atresia patients (40) who underwent hepaticoportoenterostomy had type 3 .

Bile flow was seen at the time of surgery in 35 of 43 cases of biliary atresia (81.3\%), but only $9(21 \%)$ had satisfactory response. Therefore, presence of bile flow at surgery did not indicate the outcome in this study. Irregularity or nodularity of liver was seen in 24 patients, all of them showed changes of cirrhosis which indicate a poor outcome. The histopathology examination of liver biopsy tissue showed early changes of biliary atresia in 13 (30.2\%), but only nine patients had satisfactory response.

Late changes of biliary atresia with periportal fibrosis was seen in $69.7 \%$. Of these, 6 patients had partial response and 24 therapeutic failure outcomes. The presence of cirrhosis is an important prognostic factor noted in this study. Surgery if done at an early age before the progression of hepatic fibrosis and cirrhosis would improve the outcome.

Study of milestones in post Kasai patients showed delayed psychomotor development in all patients with partial response and therapeutic failure and in two patients with satisfactory response. This reflects the malnutrition status associated with chronic liver disease.

Cholangitis was present in $37.2 \%$ of cases, which include 9 patients with satisfactory response and 7 with partial response. There were no episodes of cholangitis in patients with therapeutic failure. This observation shows that patients with satisfactory biliary drainage will develop cholangitis. This finding is in similar to study done by Carvalho et al $^{7}$ who noted ascending cholangitis in 40-60\% patient.

There was a significant decrease in bilirubin, Alanine Transaminase and Alkaline Phosphatase levels in satisfactory responders compared to a moderate decrease in partial responders and no change or further increase in the therapeutic failure group as shown in the results.

The post-operative ultrasound of abdomen showed normal appearance of liver in 6 patients belonging to the satisfactory group. While 3 patients showed mild increased echogenicity of liver among the satisfactory response. The partial response and therapeutic failure patients showed increased echogenicity, irregularity, nodularity, intrahepatic cyst formation and portosystemic collaterals suggestive of portal hypertension. Technetium 99 Mebrofenin hepatobiliary scan was done in patients with satisfactory response; 7 of the 9 cases showed complete clearance of tracer with drainage into gut by one hour. While 2 patients showed delayed clearance, even though the bilirubin level is less than $2 \mathrm{mg} / \mathrm{dL}$.

\section{CONCLUSION}

The study revealed that early surgical intervention indicated by the absence of periportal fibrosis is a good prognostic indicator. Changes in postoperative bilirubin level are a good indicator of bile drainage. The present series also emphasizes the need for regular followup, at least for a period of 5 years following portoenterostomy. By this, patient can be prognosticated and thereby refer the partial and therapeutic failure patients for early liver transplant program.

\section{SUMMARY BOX}

\section{Known Facts about the Disease}

1. Biliary atresia is a neonatal cholestatic disease.

2. This disorder represents the most common surgically treatable cause of cholestasis.

3. Encountered during the newborn period.

4. Commonest indication for liver transplantation in paediatric age group.

\section{Our Findings}

1. Biliary atresia patients operated at an early stage, that is without periportal fibrosis have satisfactory response.

2. These patients should be on regular followup.

\section{Impact on Clinical Practice in the Foreseeable Future}

1. Early surgical intervention will prevent progressive hepatocyte damage.

2. Regular follow-up will prognosticate for early liver transplantation.

\section{REFERENCES}

1. Davenport M. Biliary atresia. Seminars in Paediatric Surgery 2005;14(1):42-8.

2. Kasai M, Suzuki S. A new operation for non-correctable biliary atresia, hepatic portoenterostomy. Shujutsu 1959;13:733-9.

3. Hung PY, Chen CC, Chen WJ, et al. Long-term prognosis of patients with biliary atresia: a 25 year summary. J Paediatr Gastroenterol Nutr 2006;42(2):190-5.

4. Davenport M, de Ville GJ, Stringer MD, et al. Seamless management of biliary atresia: England and Wales 19992002. Lancet 2004;363(9418):1354-7.

5. Mohanty MK, Gupta SD, Bhatnagar V. Surgical outcome in relation to duct size at portahepatis and use of cholagogues in patients with biliary atresia. Tropical Gastroenterology 2010;31(3):184-9.

6. Sanghai SR, Shah I, Bhatnagar S, et al. Incidence and prognostic factors associated with biliary atresia in western India. Annals of Hepatology 2009;8(2):120-2.

7. deCarvalho E, Ivantes CA, Bezerra JA. Extrahepatic biliary atresia: current concepts and future directions. J Paediatr (Rio J) 2007;83(2):105-20.

8. Yanchar NL, Shapiro AM, Sigalet DL. Is early response to portoenterostomy predictive of long-term outcome for patients with biliary atresia? Journal of Paediatric Surgery 1996;31(6):774-8. 